UMTRI-2005-5

\title{
THE ROLES OF GARMENT DESIGN AND SCENE COMPLEXITY IN THE DAYTIME CONSPICUITY OF HIGH-VISIBILITY SAFETY APPAREL
}

\author{
James R. Sayer \\ Mary Lynn Mefford
}

February 2005 
THE ROLES OF GARMENT DESIGN AND SCENCE COMPLEXITY IN THE DAYTIME CONSPICUITY OF HIGH-VISIBILITY SAFETY APPAREL

James R. Sayer

Mary Lynn Mefford

The University of Michigan

Transportation Research Institute

Ann Arbor, MI 48109-2150

U.S.A.

Report No. UMTRI-2005-5

February 2005 
Technical Report Documentation Page

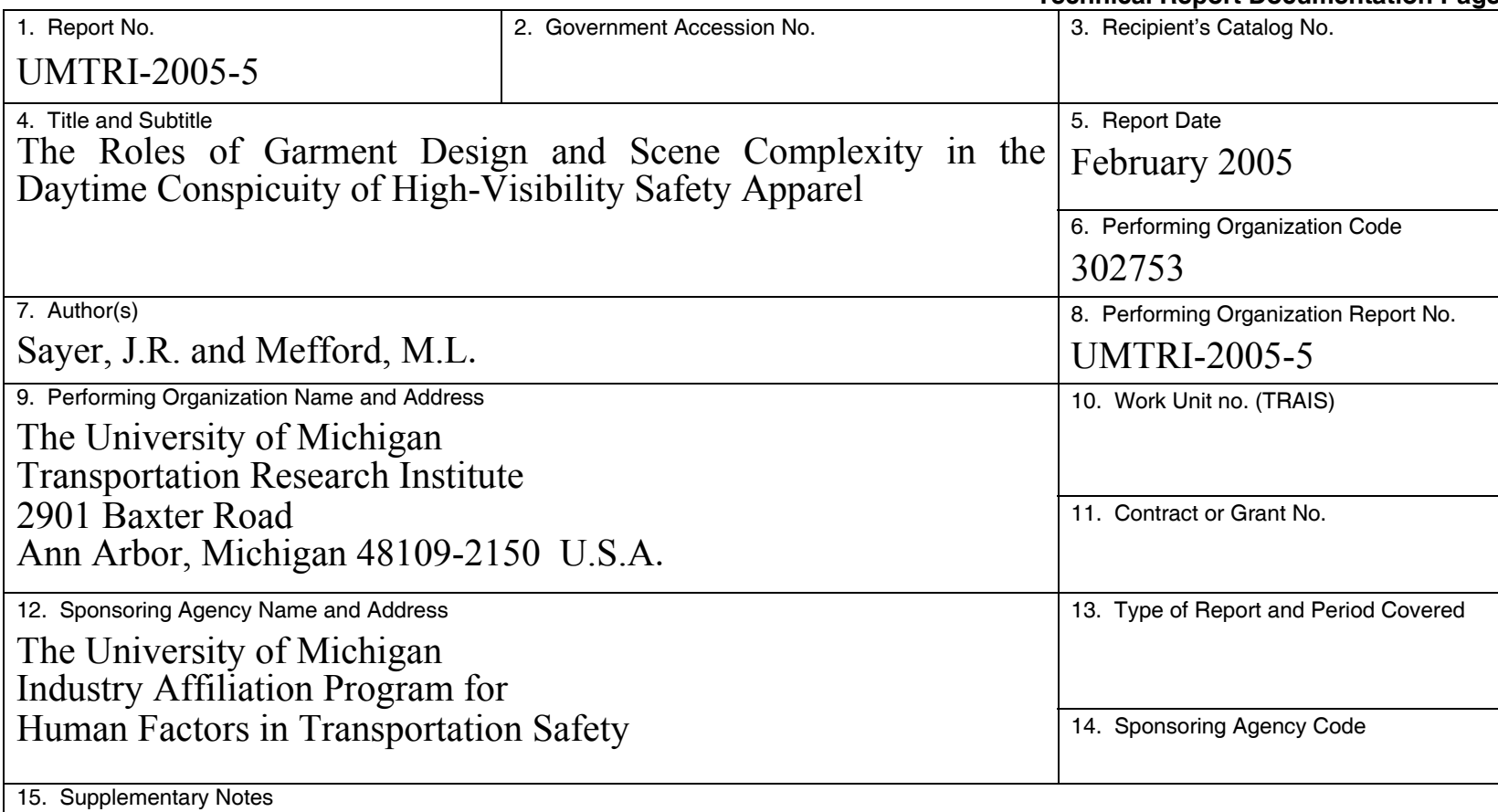

The Affiliation Program currently includes: AGC Automotive America, Autoliv, Automotive Lighting, Avery Dennison, Bendix, BMW, Bosch, DaimlerChrysler, DBM Reflex, Decoma Autosystems, Denso, Federal-Mogul, Ford, GE, General Motors, Gentex, Grote Industries, Guide Corporation, Hella, Honda, Ichikoh Industries, Koito Manufacturing, Lang-Mekra North America, Magna Donnelly, Mitsubishi Motors, Muth, Nichia America, Nissan, North American Lighting, OLSA, OSRAM Sylvania, Philips Lighting, PPG Industries, Reflexite, Renault, Schefenacker International, Sisecam, SL Corporation, Solutia Performance Films, Stanley Electric, Toyoda Gosei North America, Toyota Technical Center USA, Truck-Lite, Valeo, Visteon, 3M Personal Safety Products, and 3M Traffic Safety Systems. Information about the Affiliation Program is available at: http://www.umich.edu/ industry

16. Abstract

A naturalistic field study was conducted to assess the effects of garment color, the amount of background material, pedestrian arm motion, scene complexity, and driver age on the daytime conspicuity of personal safety garments. Sixteen drivers drove instrumented vehicles in real traffic, along a fixed $31-\mathrm{km}$ route, in search of pedestrians wearing one of four fluorescent safety garments with retroreflective trim. Distances at which the drivers first reported detecting the pedestrians were recorded. Drivers had no prior knowledge of where along the route pedestrians would be located, nor the number of pedestrians positioned along the route. All of the challenges normally encountered when driving on public roadways were present during the study (other motor vehicles, traffic signals, signs, pedestrians, and bicyclists), thus imposing an ecologically valid level of workload on the drivers.

The results show that scene complexity was the only main effects variable to significantly affect the distance at which a pedestrian wearing a fluorescent safety garment was detected. Garment color (fluorescent yellow-green or fluorescent red-orange), garment type (Class 2 vest or Class 2 jacket), arm motion (arms in motion or stationary), and driver age (younger or older) did not significantly affect the distance at which pedestrians were detected. The results contribute to a growing body of research aimed at a more general understanding of what garment characteristics enhance pedestrian conspicuity in both day and night conditions.

\begin{tabular}{|c|c|c|c|}
\hline \multicolumn{2}{|c|}{$\begin{array}{l}\text { 17. Key Words } \\
\text { Daytime, conspicuity, fluorescent, pedestrian, personal protective equipment, } \\
\text { road worker }\end{array}$} & \multicolumn{2}{|c|}{$\begin{array}{l}\text { 18. Distribution Statement } \\
\text { Unlimited }\end{array}$} \\
\hline $\begin{array}{l}\text { 19. Security Classification (of this report) } \\
\text { None }\end{array}$ & $\begin{array}{l}\text { 20. Security Classification (of this page) } \\
\text { None }\end{array}$ & $\begin{array}{l}\text { 21. No. of Pages } \\
18\end{array}$ & \\
\hline
\end{tabular}




\section{ACKNOWLEDGMENTS}

Appreciation is extended to the members of the University of Michigan Industry Affiliation Program for Human Factors in Transportation Safety for support of this research. The current members of the Program are:

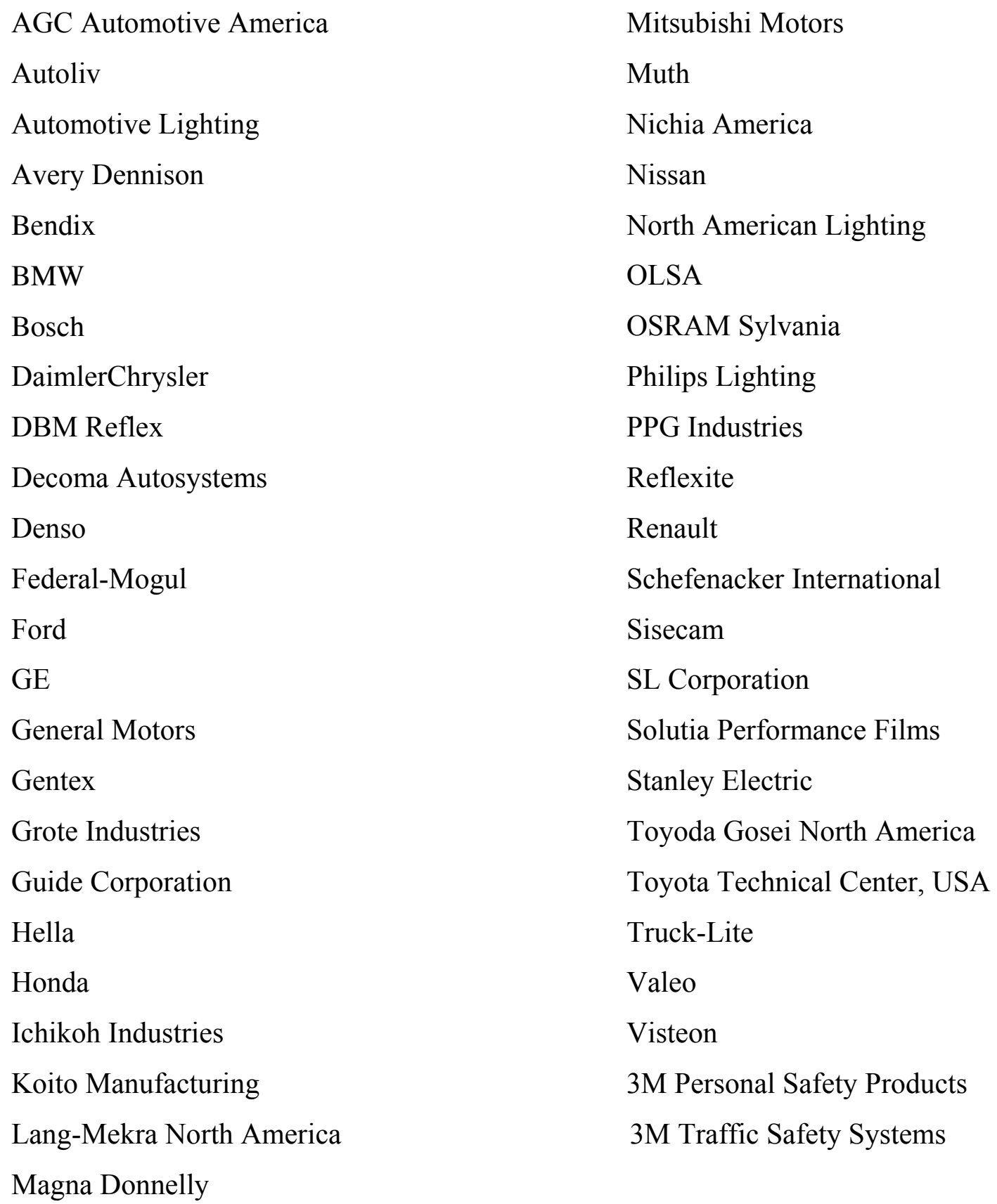

The authors would like to thank Michelle Barnes, Joel Devonshire, Chris DiVirgilio, Dillon Funkhouser, Dan Huddleson, Angelique Wetzel, Jake Wetzel, and Lisa Wiltse for their assistance in conducting this study. 


\section{CONTENTS}

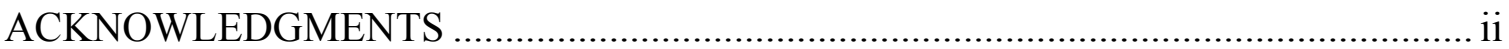

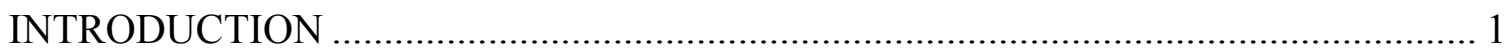

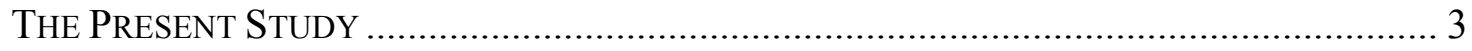

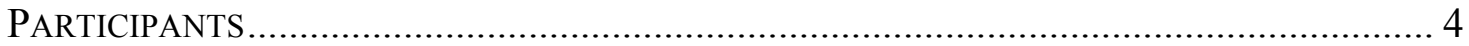

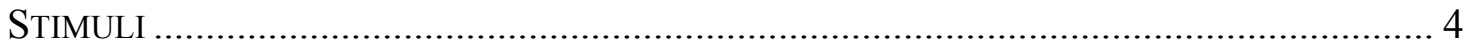

TASK AND EXPERIMENTAL SETUP.......................................................................... 5

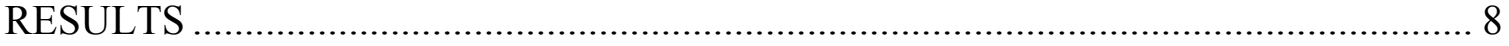

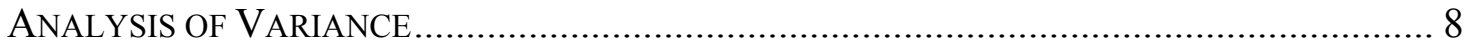

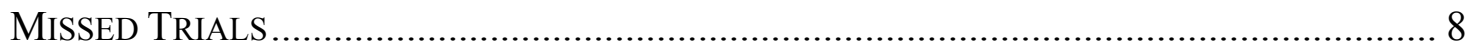

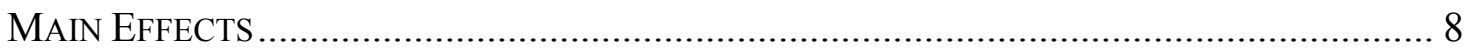

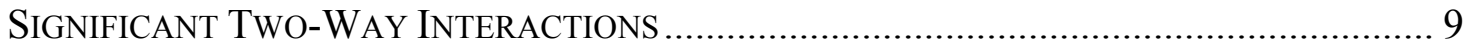

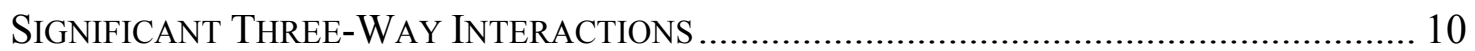

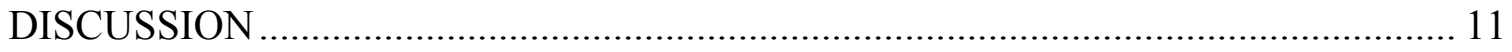

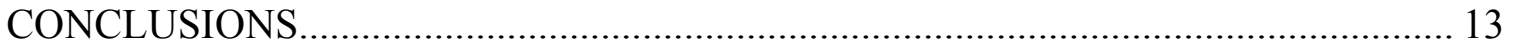

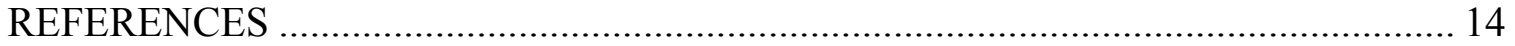




\section{INTRODUCTION}

Despite pedestrian deaths having decreased by $16 \%$ since 1993, 4,749 pedestrians were nevertheless killed in traffic crashes in the United States in 2003. Of these deaths, $35 \%$ occurred in daylight (U.S. DOT, 2004). Daytime pedestrian conspicuity, while perhaps not viewed to be as critical on the basis of fatality rates as nighttime conspicuity, nonetheless remains a serious issue-particularly as it applies to the design of highvisibility safety apparel for occupations exposed to traffic, like road construction.

The design of occupational safety apparel for road construction workers offers some unique challenges. For example, an optimal design would work well both during the day and at night. Requiring workers to wear different safety apparel for day and nighttime conditions might not only be cost prohibitive, but it may also be unrealistic in that it would lead to uncertainty in some conditions (such as at dusk or dawn) as to which garment should be worn. Use of a single garment that is suitable under all lighting conditions would mean that workers would not have to change garments as ambient lighting conditions change in order to stay safe. Additional challenges associated with the design of occupational safety apparel include providing comfortable materials that are light-weight and do not trap much heat or moisture, garments that permit worker flexibility, and durable materials that stand up to prolonged ultraviolet exposure but can be easily laundered.

The benefit of using fluorescent-colored materials in high-visibility safety garments has been recognized for many years (Michon, Eernst, and Koutstall, 1969). However, beyond the general recognition that fluorescent colors are more likely to make a pedestrian "stand out" from the surrounding scenery in daylight conditions, only a handful of research studies have examined in detail the benefits of high-visibility apparel (e.g., Ashford, Stroud, Kirkby, and Kirk, 1978), and even fewer have addressed issues

like color contrast or the amount of fluorescent materials required to optimize a garment's daytime conspicuity (e.g., Brackett and Stuart, 1982; Brich, 1998).

To make matters more complicated, road construction work zones already contain a wide variety of fluorescent orange traffic control devices that delineate the work zone from traffic. These devices can be in the form of cones, barrels, barriers, etc. In addition, 
road signs identifying the work zone or reduced speed limits within the zone, and even heavy equipment, can also be fluorescent orange. Amidst the "sea" of fluorescent orange associated with a work zone, it is increasingly challenging to make pedestrians not only detectable, but also recognizable.

A field study by Turner, Simmons, and Graham (1997) examined the effect that fabric color for vests had on detection distances in four simulated work zone settings under daytime conditions. Eleven fabric colors were examined in both cluttered and uncluttered conditions. None of the vests had retroreflective trim, but one vest was a combination of fluorescent orange and fluorescent yellow fabric. The authors reported that both vest color and work zone conditions had significant effects of detection distance, but that there was not a significant interaction of these two factors. Therefore, collapsing detection distances across work zone conditions, mean detection distances were reported for the ten vest colors and one color combination. The results showed that a fluorescent red-orange vest was detected at the longest distance. The authors concluded by recommending the use of fluorescent red-orange fabric for safety garments, even in cluttered conditions where other objects in the environment may match the color of the vest.

Turner et al. also recommended, based on their results, that fluorescent yellowgreen, or the combination of fluorescent red-orange and fluorescent yellow-green, were good alternatives to fluorescent red-orange, but that one color or color combination should be recommended for use as a national standard, and that the effect of combined retroreflective treatment with the vests should be examined under daytime conditions. However, this study was conducted on what was essentially a closed course, with little or no other traffic, and the participants were passengers in a car driven by a researcher. As such, the participants were not subjected to workload demands comparable to the conditions present in most naturalistic driving conditions, and therefore the distances at which the pedestrians were detected may not be representative of real-world conditions. In addition, only safety vests were included as stimuli in the study and only workzone scenes were examined. 


\section{The Present Study}

A naturalistic, daytime field study was conducted to assess the effects of garment color (fluorescent yellow-green or fluorescent red-orange), the amount of background material (vest or jacket), pedestrian arm motion (moving or stationary), scene complexity (low or medium complexity) and driver age (younger or older) on the conspicuity of personal safety garments. Distances at which drivers of an instrumented research vehicle detected pedestrians outfitted in the fluorescent garments were recorded. Drivers had no prior knowledge of where along a fixed $31-\mathrm{km}$ route pedestrians would be located, nor the number of pedestrians positioned along the route. All of the challenges normally encountered when driving on public roadways were present (other motor vehicles, traffic signals, signs, pedestrians, and bicyclists), imposing an ecologically valid level of workload on the drivers. 


\section{METHOD}

\section{Participants}

Sixteen paid drivers, eight older (ranging from 65 to 77 , mean age $=69.8$ ) and eight younger (ranging from 20 to 29 , mean age $=25.1$ ) took part in this study. Each of the two age groups was balanced for gender. Drivers were recruited from a list of potentially interested persons maintained by UMTRI, and each was a licensed driver. Participation required a total of three hours.

All drivers had color normal vision, as determined by using pseudoisochromatic plates (Ichikawa, Hukami, Tanabe, and Kawakami, 1978). The average visual acuity score was $20 / 23$, with younger drivers averaging better $($ mean $=20 / 19$ ) than older drivers $($ mean $=20 / 30)$. Drivers were instructed to wear any corrective lenses that they normally wear when driving during visual acuity testing, and while taking part in the study.

\section{Stimuli}

Four new ANSI/ISEA 107-2004 compliant garments were used in this study. Two of the garments had fluorescent yellow-green background material (a Class 2 vest and a Class 2 jacket) and two had fluorescent red-orange background material (a Class 2 vest and a Class 2 jacket). All garments were size XL (extra large). The vests utilized approximately $0.9 \mathrm{~m}^{2}$ of fluorescent background material, whereas the jackets used approximately $1.1 \mathrm{~m}^{2}$ of fluorescent background material. All retroreflective trim was silver, 50-mm wide, cloth-backed, exposed reflective-lenses material. Each garment contained $0.17 \mathrm{~m}^{2}$ of retroreflective trim (Figure 1).
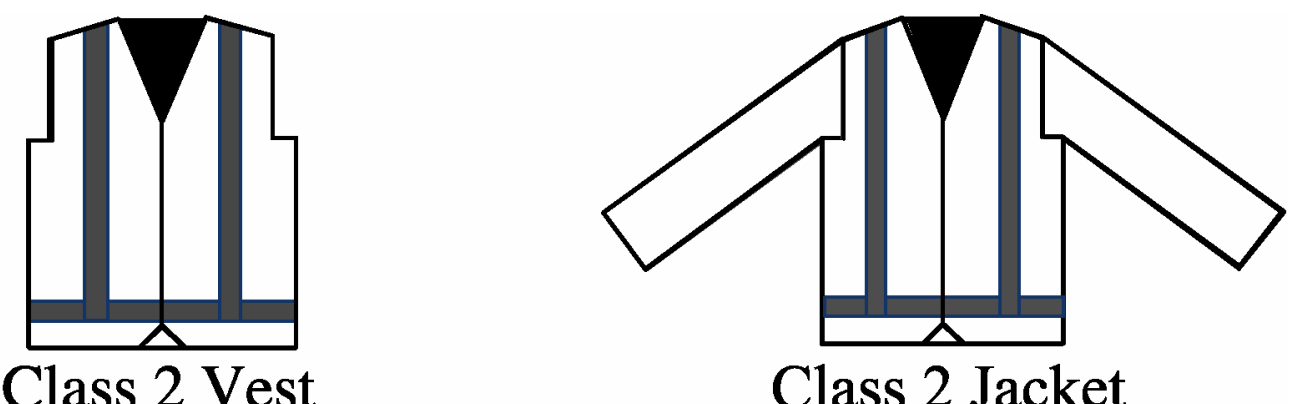

Class 2 Jacket

Figure 1. Illustration showing the types of garments used as stimuli. 


\section{Task and Experimental Setup}

The task involved performing a search task while driving the research vehicle on two traversals of a $31-\mathrm{km}$ route through Ann Arbor, MI. Drivers were instructed to indicate to an accompanying researcher when they saw a road worker wearing a highvisibility safety garment along the side of the road. Drivers were shown an example of the high-visibility safety garments they would be searching for. Drivers had no prior knowledge of where along the route pedestrians would be located, nor the number of pedestrians positioned along the route. The vehicles' windshields were regularly cleaned and testing was not performed in inclement weather. Instructions read to drivers were as follows:

Thank you for agreeing to participate in our study of conspicuity of road worker garments. You will drive the route three times and for each of the drives you will be accompanied by a researcher. The first time that you drive the route, we will not be collecting data. It's a test drive to familiarize you with the route. The researcher will serve as your navigator and will be available to answer questions that you might have about the study, but we ask that you keep any other chatting to a minimum. We would like to have you focus on driving and identifying road workers that you will see along the route. Your task is to announce, as quickly as possible, whenever you see a road worker along the side of the road by saying "Worker."

Here is an example of the vests that the workers will be wearing. (Show them the vest.) I would like for you to keep the following things in mind:

- Except for when you have been asked to make a left turn, please drive in the right lane.

- You will be given a short break between the trips.

Once again, please announce "Worker" as soon as you see a road worker along the side of a road. Please disregard any other pedestrians or bicyclists.

Do you have any questions?

Two four-door passenger cars equipped with automatic transmissions served as the instrumented research vehicles. Each vehicle was equipped with a data acquisition system which included a differential global positioning system (DGPS), a computer and hard disk, and a button used by the experimenter, positioned in the rear seat, to mark the global positioning data to indicate the location along the route where drivers first 
identified the position of the pedestrian wearing the high-visibility garment. Vehicle location was recorded in XYZ ECEF (Earth-Centered, Earth-Fixed) coordinates, and the detection distances were calculated by using the following formula:

$$
d=\sqrt{\left(x_{2}-x_{1}\right)^{2}+\left(y_{2}-y_{1}\right)^{2}+\left(z_{2}-z_{1}\right)^{2}}
$$

where $\left(\mathrm{x}_{1}, \mathrm{y}_{1}, \mathrm{z}_{1}\right)$ are the coordinates associated with where the driver saw the pedestrian, and $\left(\mathrm{x}_{2}, \mathrm{y}_{2}, \mathrm{z}_{2}\right)$ are the coordinates associated with the actual position of the pedestrian.

The study was conducted during the day on public roads in Ann Arbor. Prior to collecting data, drivers drove the route once in order to familiarize themselves with the route and to minimize the need for navigation assistance from the accompanying experimenter. All driving was done on major arterials or local roads. There were no limited access segments in the route. Experimenters posing as pedestrians were positioned along the route at sixteen different locations (eight positions per traversal). They stood on the right side of the road $1 \mathrm{~m}$ from the edge line. For each trial, an experimenter was wearing one of the four retroreflective garments and facing oncoming traffic. Additionally, in half of the trials the pedestrian's arms were stationary and in half of the trials the arms were in motion (swinging his/her arms).

Two levels of environmental complexity, low and medium, were examined. The low complexity environment was characterized by low traffic density and sparse housing/businesses (Figure 2). The activity associated with strip malls and other business areas and higher traffic densities were characteristics of the medium complexity segments of the route (Figure 3). Trials were divided evenly between the low and medium complexity environmental conditions. 


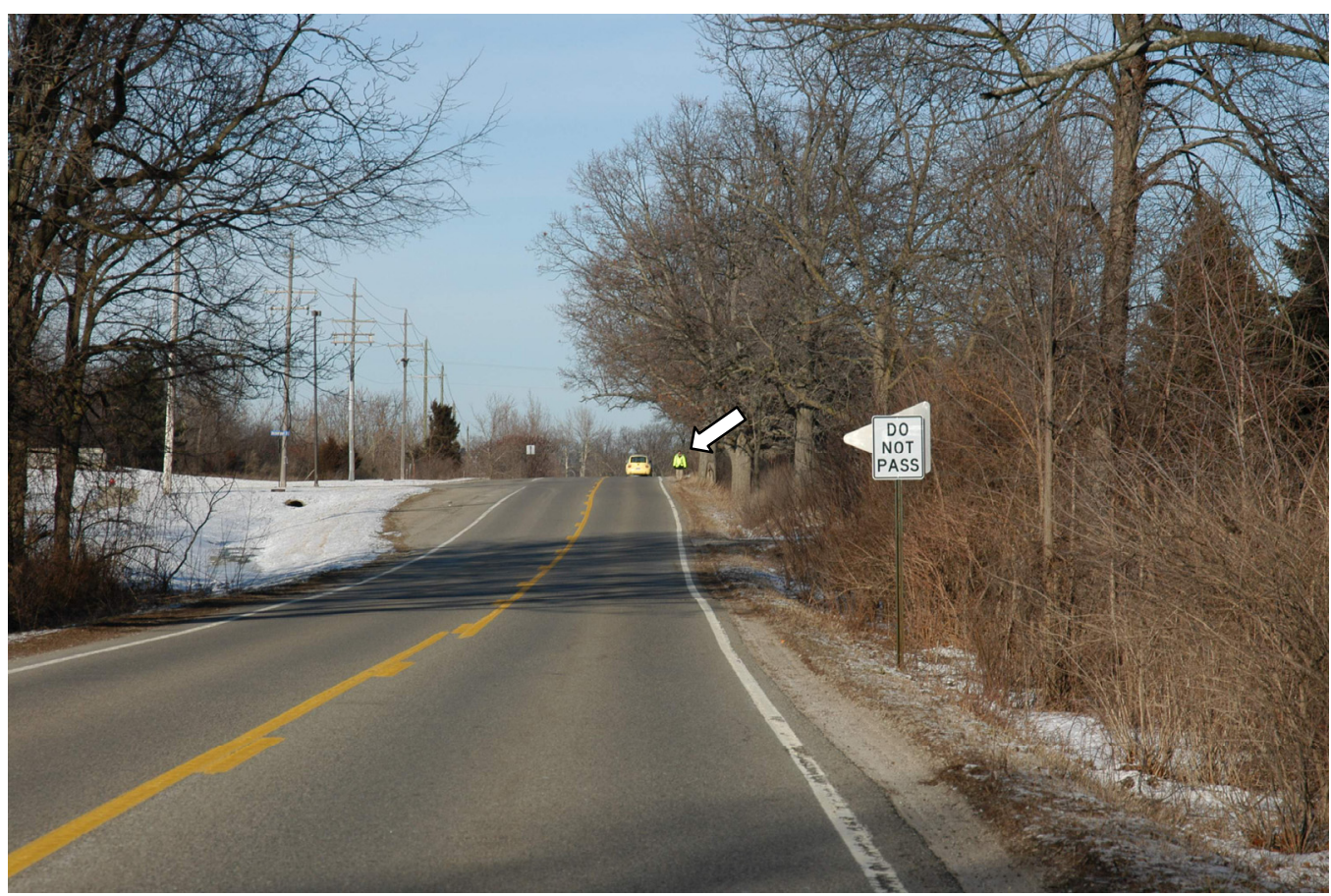

Figure 2. An example of the low complexity environment. The arrow is pointing to the pedestrian.

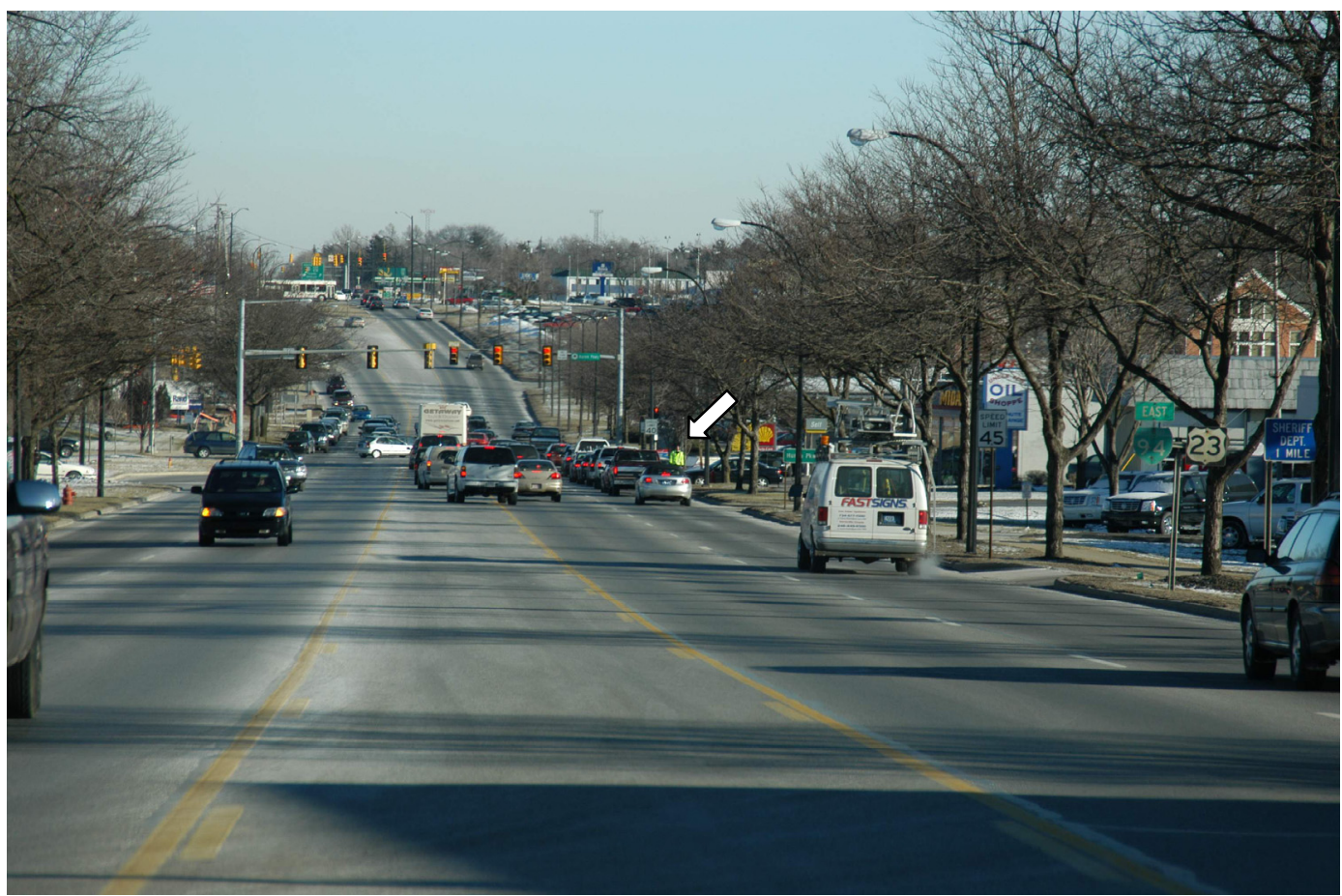

Figure 3. An example of the medium complexity environment. The arrow is pointing to the pedestrian. 


\section{RESULTS}

\section{Analysis of Variance}

A mixed-design repeated-measures analysis of variance was performed on the data. The within-subject variables were garment type (two levels), garment color (two levels), arm motion (two levels), and scene complexity (two levels). The betweensubject variable was driver age (two levels). The dependent measure was the distance at which a pedestrian was detected. The analysis included the Greenhouse-Geisser adjustment of the degrees of freedom for within-subject tests.

\section{Missed Trials}

Of the 256 trials conducted, there were four trials in which drivers missed detecting a pedestrian altogether. The four misses were associated with four different drivers. Three of the missed trials occurred when three younger drivers failed to detect a pedestrian wearing a Class 2 jacket. The remaining missed trial was for an older driver who missed a pedestrian wearing a Class 2 vest. Zero was used for detection distance on trials in which the subjects failed to detect the pedestrian.

\section{Main Effects}

Scene Complexity. There was a significant main effect of scene complexity, $F(1$, $14)=21.3, p<.001$. On average, drivers detected pedestrians $70 \mathrm{~m}$ farther in the low complexity scenes than they did in the medium complexity scenes (Figure 4). 


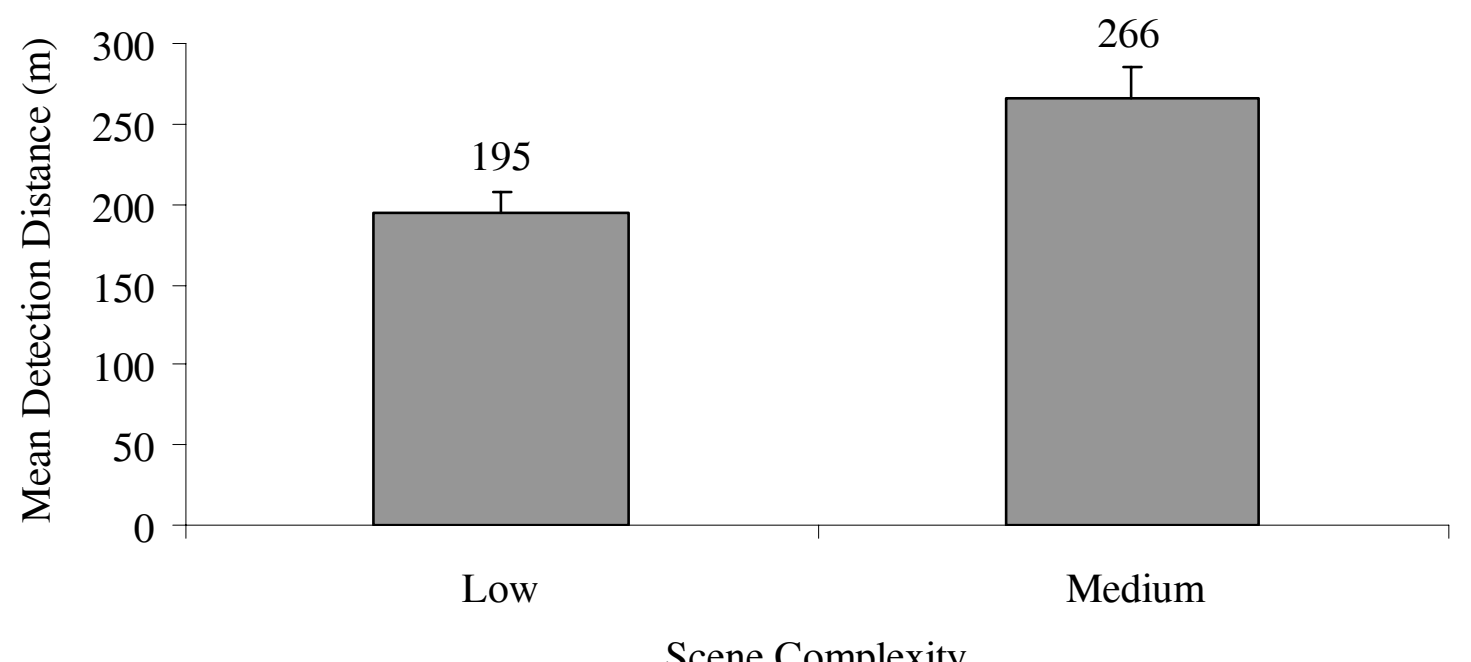

Figure 4. The main effect of scene complexity on detection distance. Error bars represent mean standard error.

The four remaining main effects were not statistically significant. While not significant, the associated mean detection distances are provided in parentheses, for reference only, after the reporting of the $F$ statistic and $p$-values. Garment type, $F(1,14)$ $=.876, p=.365$ (vest mean $=223 \mathrm{~m}$, jacket mean $=238 \mathrm{~m})$; garment color,$F(1,14)=$ $.842, p=.374$ (fluorescent orange mean $=226 \mathrm{~m}$, fluorescent yellow-green mean $=235$ $\mathrm{m}$ ); arm motion, $F(1,14)=1.2, p=.287$ (stationary mean $=225 \mathrm{~m}$, moving mean $=236$ $\mathrm{m}$ ); and driver age, $F(1,14)=1.1, p=.745$ (younger mean $=226 \mathrm{~m}$, older mean $=$ $235 \mathrm{~m})$.

\section{Significant Two-Way Interactions}

Garment by Arm Motion. Although the main effects of garment and arm motion were not significant, a significant two-way interaction between them was observed, $F(1,14)=4.9, p=.043$. Figure 5 illustrates that while pedestrians who moved their arms were on average detected at slightly farther distances when wearing the Class 2 vest $(239 \mathrm{~m})$ than when wearing the Class 2 jacket $(233 \mathrm{~m})$, pedestrians who were motionless were detected at substantially longer distances when wearing the jacket (243 $\mathrm{m})$ as compared to when wearing the vest $(208 \mathrm{~m})$. Stated another way, arm motion significantly increased detection distances, provided the pedestrian was wearing a Class 2 
vest, but arm motion had little effect on detection distances when the pedestrian was wearing the Class 2 jacket.

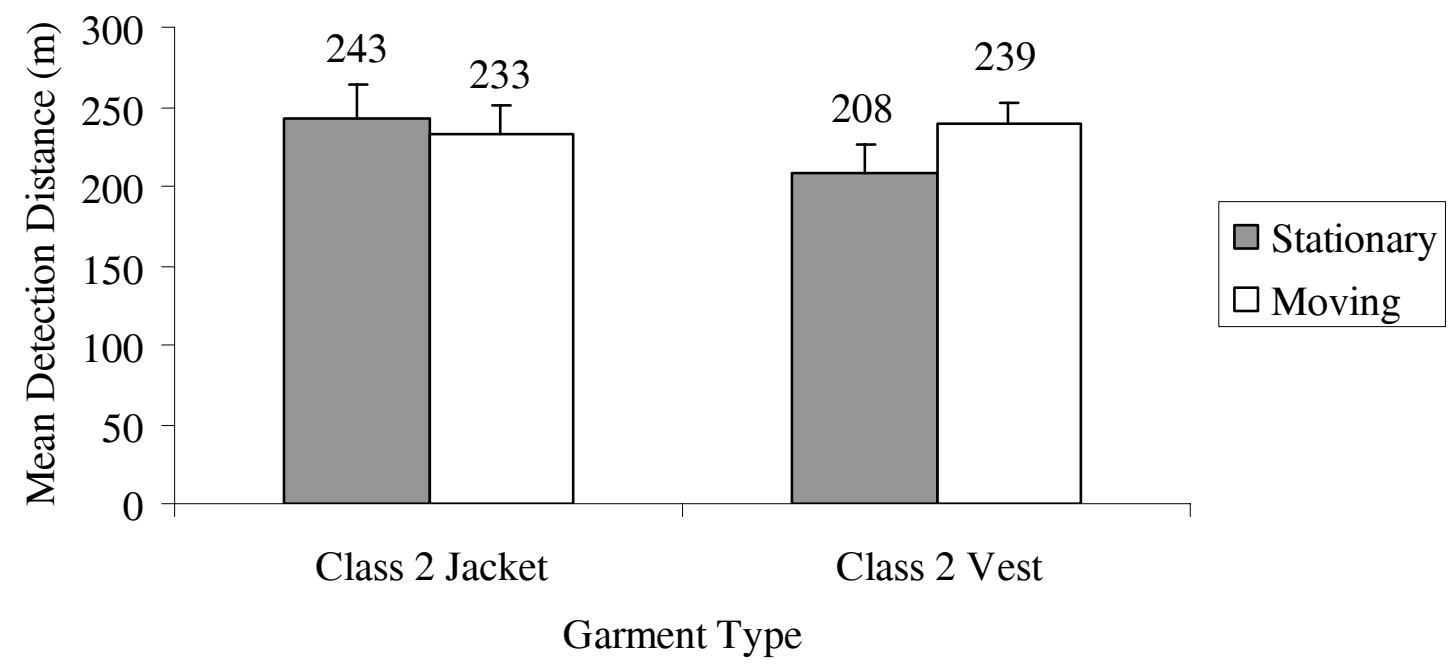

Figure 5. The interaction of garment type and arm motion on detection distance. Error bars represent mean standard error.

\section{Significant Three-Way Interactions}

There were two three-way interactions that were statistically significant, scene complexity by garment color by garment type, $F(1,14)=6.3, p=.025$, and garment color by garment type by arm motion, $F(1,14)=5.8, p=.03$. 


\section{DISCUSSION}

The only significant main effect observed in this field study was scene complexity. Specifically, pedestrians were detected at longer distances when the scene was of a lower complexity. This result is consistent with research that has been preformed under nighttime conditions where the conspicuity of retroreflectors on highvisibility garments was found to be significantly affected by the complexity of the surrounding scene (Sayer and Mefford, 2004). In both the current and previous research, the tasks have been that of search conspicuity, with the drivers having no prior knowledge of where a pedestrian might be located, but knowing that pedestrians with high-visibility garments will be present somewhere along the route. In essence, this task becomes a signal-to-noise ratio problem. The more background information a driver has to search through, or to respond to, the longer it is likely to take to locate specific targets.

Detection distances between the fluorescent yellow-green and the fluorescent redorange garments were not significantly different, nor were there any significant two-way interactions involving garment color. Consequently, to the degree that there is no color contrast incorporated into a garment either through the use of fluorescent fabric or combined-performance materials (i.e., fluorescent colored retroreflective trim), there appears to be no difference in conspicuity between the fluorescent yellow-green and fluorescent red-orange background materials. The effect on the conspicuity of safety garments incorporating contrasting fluorescent colors remains to be studied under similar naturalistic conditions. But previous research in a static setting (Sayer and Mefford, 2000) showed that the use of contrasting fluorescent colors significantly increased subjective assessments of conspicuity, and therefore warrants investigation using a more ecologically-valid methodological approach like that of the current study. Furthermore, imbedding the garments into scenes that include traffic control materials, such as roadway work zones, should also be considered. While this is reasonably straightforward to accomplish in a test-track environment (Sayer and Mefford, 2003), it is much more challenging in an ecological-valid testing procedure, like that used in the current study.

The results of this field study indicate that there was no main effect of garment type on the conspicuity of pedestrians during the daytime. The lack of a main effect for 
garment type was again similar to results reported previously in a nighttime condition (Sayer and Mefford, 2004). However, in the nighttime condition, whether a highvisibility garment included arms or not determined where retroreflective trim (the element making the garment conspicuous at night) could be placed, and not necessarily to the general conspicuity of the garment. Whereas in the daytime condition, the roles of the retroreflective trim and the background materials should have been reversed, and the fluorescent background material should be the element of the high-visibility garment most likely to make it conspicuous. However, the addition of fluorescent material in the form of the Class 2 jacket's arms did not significantly improve detection distances.

In the current study, arm motion by the pedestrian did not have a statistically significant effect on detection distances. In contrast, in the pervious study conducted at night (Sayer and Mefford, 2004), arm motion did significantly improve pedestrian detection distances, independent of the type of retroreflective treatment. Sayer and Mefford (2004) theorized that arm motion resulted in a "flashing" appearance of the retroreflectors in the nighttime condition. Because the current study was conducted during daylight hours, when the silver retroreflective trim is unlikely to contribute much to a garment's conspicuity, it is perhaps not surprising that a significant effect of arm motion was not observed. However, it is important to note that the current study only examined one type of orientation - where the pedestrian faced the oncoming vehicles. In the previous study two levels of orientation were examined, and an orientation by arm motion interaction was observed. Specifically, detection distances were shortest when the pedestrian stood motionless and perpendicular to the flow of traffic. Thus, future study related to daytime conspicuity of high-visibility garments should not necessarily exclude arm motion as a factor.

Lastly, the detection distances of high-visibility garments were not affected by driver age, even though older drivers had a somewhat lower mean visual acuity. In addition, three of the four missed trials were associated with the younger drivers and only one with an older driver. Consequently, unlike some of the previous studies examining the nighttime conspicuity of high-visibility garments incorporating retroreflectors (Sayer and Mefford, 2002), daytime conspicuity of high-visibility garments does not appear to be affected by driver age under the conditions examined. 


\section{CONCLUSIONS}

The current study was conducted under conditions that were generally more ecologically valid than those in many previous studies examining the daytime conspicuity of high-visibility garments. Consequently, the present results may be more representative of actual distances at which pedestrians wearing these garments might be detected during daylight conditions. Conducting the present study on public roads, in real traffic, provided drivers with ample workload in dealing with naturally occurring traffic and distracters (such as traffic signals, signs, pedestrians, and bicyclists).

Previous research on pedestrian conspicuity has been largely focused on dark or nighttime conditions, where pedestrian fatalities are highly overrepresented relative to the amount of pedestrian travel occurring during the nighttime hours. However, in many instances, high-visibility garments need to make pedestrians conspicuous both during the day and night. It is unrealistic to assume that pedestrians will have multiple garments at their disposal which can be readily switched due to changes in the ambient illumination, particularly around dusk and dawn, or due to changes in the complexity of the pedestrian's surroundings. Therefore, it is important to understand how the various design elements of high-visibility garments might individually, as well as collectively, contribute to pedestrian safety under a wide range of conditions. For example, the

current study found no daytime benefit of added fluorescent material contained in a jacket relative to a vest. However, that does not mean that the presence of the jacket's sleeves is not critical to the placement of retroreflective trim for nighttime conspicuity. While more remains to be learned about how the various characteristics of a highvisibility garment contribute to its conspicuity, more research, in particular, needs to be conducted during periods of transitional illumination, such as dusk and dawn. 


\section{REFERENCES}

ANSI/ISEA (2004). American national standard for high-visibility safety apparel and headwear. (ANSI/ISEA 107-2004), Arlington, Virginia: ISEA-The Safety Equipment Association.

Ashford, N., Stroud, P., Kirkby, C. and Kirk, N. S. (1978). Evaluation of motorcycle daytime visibility aids. Transportation Research Board Annual Meeting, Washington, D.C.

Brich, S. C. (1998). Development of a safety service patrol uniform standard. (Report No. VTRC 99-TAR4). Charlottesville, VA: Virginia Transportation Research Council.

Brackett, Q. and Stuart, M. (1982). High visibility garments for use in work zones. (No. NTIS PB 84 166826). College Station: The Texas A\&M University System.

Gordon, D. (1983). Design of work zone flagger's vest. (FHWA/RD-83/008). McLean, VA: Federal Highway Administration.

Harrell, W. A. (1994). Effects of pedestrians' visibility and signs on motorists' yielding. Perceptual and Motor Skills, 78, 355-362.

Michon, J. A., Eernst, J. T., and Koutstall, G. A. (1969). Safety clothing for human traffic obstacles. Ergonomics, 12(1), 61-70.

Sayer, J. R. and Mefford, M. L. (2000). The effect of color contrast on the daytime and nighttime conspicuity of roadworker vests. (Report No. UMTRI-2000-35). The University of Michigan Transportation Research Institute, Ann Arbor, MI.

Sayer, J. R. and Mefford, M. L. (2003). High-visibility safety apparel and the nighttime conspicuity of pedestrians in work zones (Report No. UMTRI-2003-29). Ann Arbor, MI: The University of Michigan Transportation Research Institute.

Sayer, J. R. and Mefford, M. L. (2004). The roles of retroreflective arm treatments and arm motion in nighttime pedestrian conspicuity. (Report No. UMTRI-2004-21). The University of Michigan Transportation Research Institute, Ann Arbor, MI. 
Turner, J. D., Simmons, C. J., and Graham, J. R. (1997). High-visibility clothing for daytime use in work zones. In Safety and Management in Maintenance and Construction Operations, Transportation Research Record No. 1585, Washington, D.C.: Transportation Research Board, National Research Council.

U. S. Department of Transportation (2004). Traffic safety facts 2003: Pedestrians. Report DOT HS 809 769, National Center for Statistics and Analysis, National Highway Traffic Safety Administration. Washington, DC. 